\title{
Compressed sensing image restoration algorithm based on improved SURF operator
}

https://doi.org/10.1515/phys-2018-0124

Received October 9, 2018; accepted November 14, 2018

\begin{abstract}
Aiming at the excellent descriptive ability of SURF operator for local features of images, except for the shortcoming of global feature description ability, a compressed sensing image restoration algorithm based on improved SURF operator is proposed. The SURF feature vector set of the image is extracted, and the vector set data is reduced into a single high-dimensional feature vector by using a histogram algorithm, and then the image HSV color histogram is extracted.MSA image decomposition algorithm is used to obtain sparse representation of image feature vectors. Total variation curvature diffusion method and Bayesian weighting method perform image restoration for data smoothing feature and local similarity feature of texture part respectively. A compressed sensing image restoration model is obtained by using Schatten-p norm, and image color supplement is performed on the model. The compressed sensing image is iteratively solved by alternating optimization method, and the compressed sensing image is restored. The experimental results show that the proposed algorithm has good restoration performance, and the restored image has finer edge and texture structure and better visual effect.
\end{abstract}

Keywords: SURF operator, compressed sensing, image restoration

PACS: 07.05.Kf, 07.05.Pj, 07.05.Tp

\footnotetext{
^Corresponding Author: Guodong Zhou: College of Mechanical and Electrical Engineering, Central South University, Changsha, 410004, China; Department of Mechanical and Electrical Engineering, Hunan Radio and TV University, Changsha, 410004, China, E-mail: smky002@126.com

Huailiang Zhang: College of Mechanical and Electrical Engineering, Central South University, Changsha 410004, China Raquel

Raquel Martinez Lucas: Department of Mathematics, University of a Castilla La Mancha, 16071-Cuenca, Spain
}

\section{Introduction}

With the rapid development of computer networks and multimedia technologies, the number of images is growing rapidly, and the demand for efficient browsing of images is increasing [1-6]. Therefore, content-based image classification and retrieval technology is a hot topic at present, but the images are diverse and most of them the image is affected by illumination, noise, size and position of the object, which increases the difficulty of image classification, so it is important to accurately acquire the stable features of the image [7-14]. The SURF operator can accurately describe the local texture features of the image and provide an effective technical means for image feature extraction [15-18]. Human vision is an extremely important means of perception for human beings. About $70 \%$ of the information people gotten is from vision [19-21]. Images are the material representation of human perception of vision, so images become an important way for people to understand the external world and themselves [4]. Since the image was taken, image restoration came into being [7]. Image restoration technology was first applied to the restoration of medieval art in the Renaissance, and trained professionals filled the cracks to restore the original image [6]. Among them, the technique of filling the damaged area such as cracks and scratches in the damaged image to make it try to restore the original appearance is called image restoration or retouching. Image restoration is an image processing technique that uses the information already in the image to repair or remove the damaged area. The purpose is to improve the visual effect of the image [12]. The repair of a partially broken image is a process that uses some prior knowledge of the image to reconstruct or restore the original image as much as possible [12].

Literature [9] proposed a compressed sensing image restoration method based on rank minimization. The compressed sensing image restoration problem was studied as a low rank matrix recovery problem, and the non-local similarity model was sampled. The similar image block was used as the column vector to construct a $2 \mathrm{D}$ similar blocks matrix, and to guarantee the low rank attribute 
of the matrix according to the strong correlation between the column vectors; Using the compressed sensing measurement as a constraint condition, the obtained 2D similar block matrix was solved by low rank matrix recovery; In the process of algorithm solving, the augmented Lagrangian method was used to transform the constrained optimization problem into an unconstrained optimization problem. The linearization technique based on Taylor expansion was used to accelerate the algorithm, complete the low rank matrix recovery, and then achieve compressed sensing image restoration. The convergence rate of the algorithm is good, but the computational complexity is high, and the needed storage space is too large. In literature [14], an image fusion algorithm based on non-downsampling double-tree complex contour transform and compressed-sensing pulse coupled neural network was proposed. Firstly, the source image was decomposed by NSDTCT to obtain the low frequency sub-band and high frequency sub-band coefficients. For the low frequency sub-band coefficients, an adaptive weighted fusion rule based on the combination of regional average gradient, region energy and S function was proposed; for the high frequency sub-band coefficients, the fusion rules based on CS-PCNN theory were proposed. The improved Laplacian energy was used as the external input of PCNN. Finally, the fusion coefficient was inversely transformed by NSDTCT to obtain the fused image. This method can effectively improve the quality of the image, but the reconstruction effect is poor.

\section{Methods}

\subsection{SURF feature vector extraction and image color feature extraction}

\subsubsection{Feature point detection}

In order to find feature points in different scale spaces, SURF establishes the scale space of the image [3]. For a 2D image $I(x, y)$, the image is convolved with the Gaussian kernel to obtain a space $L(x, y, \sigma)$ with different scales as:

$$
L(x, y, \sigma)=I(x, y) \star G(x, y, \sigma)
$$

Where, $G(x, y, \sigma)$ is a Gaussian function with variable scale.

In order to improve the computational efficiency, SURF uses rectangular filtering instead of second-order Gaussian filtering when establishing the scale space. The size of the rectangular filter is changed instead of the size of the image to obtain the scale space [5], and the integral image is used as an intermediate to accelerate the convolution operation [10].

The SURF operator uses the Hessian matrix to detect extreme points. For a point $X=(x, y)$ in image $I$, the Hessian matrix $H(X, \sigma)$ of the point at scale $\sigma$ is:

$$
H(X, \sigma)=\left[\begin{array}{cc}
L_{x X}(X, \sigma) & L_{x y}(X, \sigma) \\
L_{x y}(X, \sigma) & L_{y y}(X, \sigma)
\end{array}\right]
$$

Where $L_{x x}(X, \sigma)$ indicates that the Gaussian first-order partial derivative is convolved with image $I$ at $X$, and $L_{y y}(X, \sigma)$ and $L_{x y}(X, \sigma)$ are similar; $\sigma$ indicates the scale value at which the feature point is located.

In order to accurately approximate the Gaussian kernel function, the $H$ matrix discriminant is:

$$
\operatorname{det}\left(H_{\text {approx }}\right)=D_{x x} D_{y y}-\left(w D_{x y}\right)^{2}
$$

Among them, $D_{x x}, D_{y y}$, and $D_{x y}$ respectively represent the approximation of $L_{x x}(X, \sigma), L_{y y}(X, \sigma)$, and $L_{x y}(X, \sigma)$ after using a rectangular filter, $w$ is a weight, and is usually 0.9 .

After using the Hessian matrix to find the extremum, the non-maximum suppression is performed in the $3 \times 3 \times 3$ stereo neighborhood, only 9 of the upper and lower scales and 8 of the 26 neighborhoods around the scale are both large or small extreme points, they can be used as candidate feature points [13], and then interpolated in scale space and image space to obtain stable feature point positions.

\subsubsection{Feature point descriptor}

When describing the feature point, its main direction should be firstly determined. In order to ensure the rotation invariance of the operator, firstly, with the feature point as the center, the Haar wavelet response with the side length of 6 in the $x$ and $y$ directions of the neighborhood with the radius of $6 \sigma$ is detected, and then Gaussian weight coefficients are assigned to these response values, so that the response contribution to the feature points is large, thereby obtaining a series of vectors; Then a new vector with the sum of the responses in the range of $60^{\circ}$ is calculated, and the window is sided in unit of $60^{\circ}$, to traverse the entire circular area [2], and select the longest vector direction as the main direction of the feature points.

After determining the main direction of the feature point, the coordinate axis is firstly rotated to the main direction centering on the feature point, and a square area with a side length of $20 \sigma$ is selected. The descriptor win- 
dow is divided into $4 \times 4$ sub-regions, and within each subregion, the Haar wavelet response in the range of $25 \sigma$ is calculated, and the Haar wavelet responses in the horizontal and vertical directions with respect to the main direction are respectively recorded as $d_{x}$ and $d_{y}$. Then Gaussian weights are assigned to these responses, and finally the response values of each sub-region and the absolute values of the responses are added to generate the feature vector $V^{\prime}$ of the descriptor (as shown in equation (4)). The 4-dimensional vector is described as a feature of a subregion. Each feature point is composed of $4 \times 4$ sub-regions, and a 64-dimensional vector is formed. Finally, the vector is normalized to make it robust to illumination.

$$
V^{\prime}=\left(\sum d_{x}, \sum d_{y}, \sum\left|d_{x}\right|, \sum\left|d_{y}\right|\right)
$$

\subsubsection{Data reduction of random histogram for SURF feature vector set}

The SURF feature vector set of the image can be extracted by the above method, and each feature vector is 64-dimensional. Generally, the SURF feature points of an image are hundreds or even thousands. The similarity between the two images is determined by the number of SURF feature points matched by the two images, if the ratio of the nearest neighbor distance to the next nearest neighbor is less than the threshold, then the two points are matched successfully, otherwise there is no matching point; If the exhaustive method is used for direct matching, that is, the distance between the eigenvectors of the two images is exhausted, the time complexity is high and the time is long. Moreover, the SURF feature extracts a vector set and cannot be directly classified by a classifier such as SVM.

According to the above analysis, a random histogram algorithm is proposed to transform the SURF feature vector set into a single higher-dimensional feature vector, that is, a random histogram to represent the original feature set according to the distribution of SURF feature points. The feature vectors thus obtained can be directly classified by the SVM classifier. The algorithm for a random histogram is:

$n$ feature points are extracted for each image, each feature point is 64-dimensional. For each feature point, $B$ bits are randomly selected, and a binary array of $B$ bits is obtained by projecting to the Hamming space according to the random threshold vector, and it is converted into a decimal number as a hash value. If the feature vectors are similar, the probability of the hash value is the same that is large.
A vector with a length of $2^{B}$ and an initial value of zero is constructed. 1 is added to the corresponding bit of the vector according to the hash value. For example, if the decimal number is 76 , then to add 1 to the corresponding 76 th bit.

In order to reduce the error, steps 2.1.1 and 2.1.2 are repeated $M$ times, and $M$ vectors are superimposed by corresponding bits to generate a $2^{B}$-dimensional vector.

In order to reduce the error, the above whole process is repeated $N$ times, and the obtained $N$ vectors are combined to obtain a $2^{B} \times N$-dimensional vector, and finally the proportion of each component is calculated, that is, a onedimensional histogram of the image $2^{B} \times N$ is obtained.

\subsubsection{Extraction of color features}

The global features of the image include features such as color, texture, shape and space, while the color features are the most reliable and stable visual features.Compared with other visual features, color features do not affect the size, position and direction of the image, and have a certain degree of robustness, and the calculation of color eigenvalues is simple, so color features are widely used in image restoration process [10].

In all the color spaces of the image, each component of the HSV color space directly acts on the visual experience, it is a uniform color space, which can better reflect people's perception of color, and is very suitable for colorbased image retrieval. Color attributes such as Hue, Saturation and Value are used by HSV to represent the color signal. The hue indicates different colors, the saturation indicates the depth of the color, and the brightness indicates the brightness of the color, which are influenced mainly by the intensity of light [1].

Since the color of an image is very large, the dimension of the histogram vector is very large, so the HSV space must be properly quantized before the histogram is calculated. The three components $H, S$, and $V$ are quantized at different intervals according to people's perception of color. The specific steps are as follows:

According to the human visual resolution, the hue space $H$ is divided into eight sections, and the saturation space $S$ and the luma space $V$ are respectively divided into three intervals.

Non-equal interval quantization is performed based on different ranges of colors and subjective color percep- 
tion. The formula for quantification is:

$$
\begin{gathered}
H=\left\{\begin{array}{l}
0, h \in[316,20] \\
1, h \in[21,40] \\
2, h \in[41,75] \\
3, h \in[76,155] \\
4, h \in[156,190] \\
5, h \in[191,270] \\
6, h \in[271,295] \\
7, h \in[296,3155]
\end{array}\right. \\
S=\left\{\begin{array}{l}
0, s \in[0,0.2] \\
1, s \in[0.2,0.7] \\
2, s \in[0.7,1]
\end{array}\right. \\
V=\left\{\begin{array}{l}
0, v \in[0,0.2] \\
1, v \in[0.2,0.7] \\
2, v \in[0.7,1]
\end{array}\right.
\end{gathered}
$$

The three color space components are combined into one-dimensional feature vectors according to the quantization level of the above equation:

$$
Q=9 H+3 S+V
$$

In this way, the three components $H, S$, and $V$ are distributed on the one-dimensional vector. The equation (8) fully and subtly applies the color information of the image, which reduces the influence of brightness and saturation on the image classification result. It can be seen that the range of $Q$ is $[0,1, \ldots, 71]$, and the proportion of the various color components on $Q$ is calculated, that is, the one-dimensional color histogram of the image 72 handle is obtained.

\subsection{Local restoration of image's structure part and texture part}

According to the visual principle, natural images can generally be decomposed into structure parts and texture parts. Image texture is a quantitative description of the uneven grooves or patterns in the image, which can provide detailed information such as spatial layout and brightness of the image. The structue part of the image refers to the segmented smooth part of the image component, which is visually expressed. In visual expression, it is the dividing line or edge of different areas in the image [10].

Among the many image decomposition algorithms, the decomposition algorithm based on DCT and the decomposition algorithm based on the bounded variational space are greatly influenced by the image restoration domain. According to prior knowledge, DCT transform can be used to decompose the image into high frequency and low frequency parts, corresponding to the texture and structure parts in the image respectively. It can also use the minimum solution of BV space to decompose the image into texture and structure.

The traditional method considers that any natural image can be decomposed into two parts: texture and structure. Assuming that the original image is $I(x, y)$, the image structure part is $u(x, y)$, and the texture part is $v(x, y)$. The original image $I$ can be expressed as:

$$
I(x, y)=u(x, y)+v(x, y)
$$

To prove the feasibility of the model, the algorithm uses an oscillation function to extract the texture part $v$ of the image, and extracts the structure part $u$ from the image using the minimization of the bounded variation space. Among them, the equation for structure part decomposition of the image is:

$$
\inf \left\{F(u)=\int\|\nabla u\|+\lambda|v|^{2}, I=u+v\right\}
$$

In the equation, $\lambda$ is the adjustment parameter. In the first term, the variation is used to remove the interference of the noise in the damaged image to the repair process, and the second term is the exact term to reduce the error between the original image $I$ and $u$.

The parameter $\lambda$ can be used to remove the texture part of the image. When $\lambda$ is close to 0 , the image $F(u)$ obtained by the equation (10) is the structure part of the original image. At this point, the decomposition algorithm essentially uses the total variation method to decompose the structure part from the original image. In the specific implementation, the traditional method treats the BV space method of the extracted structure part and the oscillation function method of the texture part as a double-BV space method. The image texture part model is given by:

Assuming that $G$ represents a Banach space consisting of a generalized function $v(x, y)$, where $v(x, y)$ can be expressed as:

$$
v(x, y)=\partial_{x} g_{1}(x, y)+\partial_{y} g_{2}(x, y)
$$

Equation (11) is derived from norm $\|I\|$, and can be defined as the lower bound of all $L^{\infty}$ norms of the function $|g|$. Where $|g|=\left(g_{1}, g_{2}\right)$, and the lower bound can be obtained by calculating all generalized functions $v$ by $I$ decomposition. It is pointed out in the conventional method that the Banach space $G$ at this time is the BV space corresponding to the texture part.

If $v$ is used to represent the texture part of the image, then $v$ is in space $G$, then the decomposition model of the 
image is:

$$
\inf \left\{\mathrm{E}(\mathrm{u})=\int|\nabla u|+\lambda\|v\|\right\}
$$

In summary, the arbitrary natural image $I$ can be decomposed into the structure part $u$ and the texture part $v$ by the equation (12). The texture information thus decomposed includes not only the uneven groove part of the image but also the brightness information of the dividing line of the different parts of the image.

A step-by-step image restoration method that is decomposed by MCA due to the above image decomposition idea. The method first decomposes the damaged image and then repairs it separately in a targeted manner.

Commonly used image restoration algorithms mainly include partial differential equation based restoration algorithms and texture filling based image restoration algorithms. In different application scenarios, the two algorithms exhibit different repair characteristics. The image restoration method based on partial differential equations has a very detailed theoretical basis and has a good restoration effect on image damage in a small area. However, since the image restoration algorithm based on partial differential equations uses pixels as the basic processing unit and restores along the damaged edge by means of information diffusion, this method is based on the structural information of the image, and the amount of information used is limited, so it only can restore the smooth part of the damaged image very well, and can't process the texture information of the image. The texture-filling-based image restoration algorithm is developed by the texture synthesis method. It is inspired by the traditional method of image decomposition theory. Combined with the experience of the overall structure first and the local texture second during manual restoration, the step-by-step image restoration method of MCA decomposition is cited in this paper, in order to introduce compressed sensing technology into image restoration technology. The design idea of the algorithm is to first use the MCA decomposition to decompose the image into two parts: texture and structure, and then perform partial restoration on the two parts obtained by the decomposition. The algorithm uses the full-variation curvature diffusion method in the wavelet domain to restore the image structure part, and uses the Bayesian weight restoration method to locally restore the texture part.

According to the above design ideas, the algorithm is mainly composed of three parts: image decomposition, restoration of texture part and restoration of structural part.

The restoration algorithm proposed in this paper uses MCA to complete the decomposition of the image. After the image is decomposed into two parts: structure and texture, the smooth information in the image is only included in the structure image, while the more important image details in human vision are both basically in the texture image.

When the image is restored, the restoration algorithm does not need to consider too many texture factors. The full-variation curvature diffusion model can be used to restore the structure image. In the specific restoration, the algorithm converts the image to the wavelet domain and then restores it. This processing can improve the robustness of the algorithm to noise in the image.

For the restoration of the texture part, although the texture restoration algorithm has a better repair effect on the large area of the damaged region, the specific selection of the target fill texture only takes into account the local information of the damaged area, and more texture blocks will appear the wrong filling phenomenon in actual use. In this paper, the Bayesian weight model is used to restore the texture part. When the texture part is restored, the local and global information is considered comprehensively, and the appropriate information block is selected to restore the damaged area. Therefore, the restoration algorithm in this section performs the texture part image.

\subsubsection{Image decomposition}

In the restoration algorithm proposed in the paper, it is assumed that the input damaged image is $X$, which is a matrix of $N \times N$, and a one-dimensional vector of length $N^{2}$ of $X$ can be obtained by matrix rearrangement. Assuming that the damaged image $X$ contains a texture, which is represented by $X_{t}$. According to the sparse representation principle of compressed sensing, an overcomplete dictionary $T_{t}$ can be used to obtain a sparse representation $a_{t}$ of $X_{t}$, where $X_{t} \in N^{2} \times L$.

$$
a_{t}^{o p t}=\arg \min \|a t\|_{0} s, t, X_{t}
$$

Where $a_{t}$ is the sparse coefficient of the texture part $X_{t}$ in the overcomplete dictionary $T_{t}$, where $T_{t}$ is required to have good adaptability to the sparse representation of different texture images. $\left\|a_{t}\right\|_{0}$ is the $\ell_{0}$ norm of vector $a_{t}$, and the meaning is the number of non-zero numbers in $a_{t}$.

In addition to being able to have a good sparse representation of the texture part, the decomposition algorithm also requires that the representation of overcomplete dictionary $T_{t}$ for non-textured images is non-sparse. That is to say,if an imagecontaining only segmentation smoothing information uses a $T_{t}$ transform, and $\left\|a_{t}\right\|_{0}$ of the obtained coefficient $a_{t}$ is close to $L$, then the representation 
$a_{t}$ at this time is non-sparse. Therefore, the dictionary $T_{t}$ can be used as a tool for distinguishing different parts of the texture and structure in the image, and the texture part in the image can be obtained by $T_{t}$.

$$
T_{t}^{o p t}=\arg \min \frac{\sum_{k}\left\|a_{t}^{o p t}(k)\right\|_{0}}{\sum_{j}\left\|a_{t}^{o p t}(j)\right\|_{0}}
$$

Where, in equation (14),

$$
\begin{gathered}
a_{t}^{o p t}(k)=r g \min \left\|a_{t}\right\|_{0} s, t, X_{t}(k) \\
a^{o p t}(k)=r g \min \|a\|_{0} s, t, X_{t}(j)
\end{gathered}
$$

The above equation indicates that the effect of $T_{t}$ is to measure the relative sparsity of the texture image in its corresponding natural image, i.e., the texture part $X_{t}$ can be extracted from the natural image $X$ using the dictionary $T_{t}$.

Similar to the above requirements, the overcomplete dictionary $T_{n}$ is defined so that it can only be used for the sparse representation of the segmented smooth structural part $X_{n}$ in the image, and the representation of the texture image $X_{t}$ is non-sparse. That is, the structural part $X_{n}$ in the image can be extracted by $T_{n}$.

For any natural image $X$ containing textures and structural parts, it can be converted to a used complete dictionary $T_{t}$ and $T_{n}$ representation:

$\left\{a_{t}^{\text {opt }}, a_{n}^{\text {opt }}\right\}=\arg \min \left\|a_{t}\right\|_{0}+\left\|a_{n}\right\|_{0} s, t, X=T_{t} a_{t}+T_{n} a_{n}$

It is shown in the equation (17) that the damaged image $X$ can be represented as the structural part $T_{n} a_{n}$ and the texture part $T_{t} a_{t}$ using the overcomplete dictionary $T_{n}$ and $T_{t}$. The condition for this decomposition is that the structure dictionary $T_{n}$ can only effectively represent the structural part of the image, and cannot be used to represent the texture part of the image, and vice versa for the texture dictionary $T_{t}$.

Since solving the $\ell_{0}$ norm problem is a non-convex problem, it is difficult to use computer operations. As the number of columns in the dictionary increases, the computational complexity of the equation grows exponentially. In the process of solving the basic tracking (BP) method, the $\ell_{1}$ norm is used to replace the $\ell_{0}$ norm, and the solution of the problem is transformed into the optimal approximation. In this case, the above decomposition model becomes:

$\left\{a_{t}^{\text {opt }}, a_{n}^{\text {opt }}\right\}=\arg \min \left\|a_{t}\right\|_{1}+\left\|a_{n}\right\|_{1} s, t, X=T_{t} a_{t}+T_{n} a_{n}$

When the sparsity of $a_{t}^{o p t}$ and $a_{n}^{o p t}$ is large, the base tracking can obtain more accurate results. In the process of performing specific decomposition calculations, there are two issues to be aware of.

The first question is that noise in the damaged image may affect the decomposition of the image, causing the image information not to be well represented by $T_{n}$ and $T_{t}$. This may result in the coefficients $a_{t}^{o p t}$ and $a_{n}^{\text {opt }}$ obtained by the decomposition being not sparse, resulting in failure of image decomposition. For this problem, a relaxation term can be introduced to make the decomposition image not strictly the same as the original damaged image, but approximately the same, increasing the robustness of the algorithm to noise. The relaxation term is introduced into equation (18), and the decomposition model becomes:

$\left\{a_{t}^{\text {opt }}, a_{n}^{\text {opt }}\right\}=\arg \min \left\|a_{t}\right\|_{1}+\left\|a_{n}\right\|_{1}+\lambda\left\|X-T_{t} a_{t}+T_{n} a_{n}\right\|$

In the equation (19), $\lambda\left\|X-T_{t} a_{t}+T_{n} a_{n}\right\|$ indicates that the image is neither a texture part nor a structural part. Introducing the slack term in this way not only ensures that the decomposition algorithm extracts the structure and texture parts from the damaged image, but also removes the additive noise that may exist in the image. The $\ell_{2}$ norm is used in equation (19) to simulate additive white Gaussian noise.

The second question is how to choose the right overcomplete dictionary. In practical applications, it is difficult to find a dictionary that satisfies the corresponding conditions for all texture images. Therefore, for different types of images, you may need to choose a different decomposition dictionary. A large number of experimental results show that for most images, the local discrete cosine transform can be used in the decomposition to obtain the texture part of the image, and the curvelet can be used as a dictionary of structural parts.

In addition, because the structural part can largely reflect the image's visual information feature, how to accurately obtain the sparse representation of the structural part is very important. This requires the structure dictionary $T_{n} a_{n}$ to have good adaptability to various types of images. Since the total variation method can well adapt to the segmentation smoothing part of the image, the total variation adjustment is added when the algorithm is implemented:

$$
\begin{aligned}
& \left\{a_{t}^{o p t}, a_{n}^{o p t}\right\}=\arg \min \left\|a_{t}\right\|_{1}+ \\
& \left\|a_{n}\right\|_{1}+\lambda\left\|X-T_{t} a_{t}+T_{n} a_{n}\right\|+\gamma T V\left\{T_{n} a_{n}\right\}
\end{aligned}
$$

Where, $\gamma \operatorname{TV}\left\{T_{n} a_{n}\right\}$ is the total variation adjustment. Since the total variation model can express the structural part well, $T_{n} a_{n}$ calculated by the decomposition algorithm added to the adjustment item can be used to associate the structural part of the image. 
So far, the input damaged image $X$ can be decomposed into the texture part $X_{t}$ and the structural part $X_{n}$ by the algorithm described above.

\subsubsection{Restoration of structural parts}

This section will focus on how to use the full-variation curvature diffusion algorithm in the wavelet domain to perform damage on the structural part. Because the TV model can not repair the damage of large curvature fracture well, the restoration algorithm of the structural part takes the curvature into consideration when selecting the information diffusion direction, and improves the restoration effect of the damaged area of the block. Let the structure image's damage area be $\Omega$, and for the structure image $X_{n}$, it can use the following model to describe:

$$
X_{n}(x)=X_{n 0}(x)+n(x)
$$

In the equation, $X_{n 0}(x)$ is an image after denoising of $X_{n}$, and $n(x)$ is additive white Gaussian noise.

The prototype of the wavelet transform is:

$$
X_{n 0}(a, x)=\sum_{j, k} a_{j, k} \varphi_{j, k}(x)
$$

For the multi-scale wavelet transform based on tensor product in 2D space, three letter wavelets of $\varphi^{1}, \varphi^{2}$ and $\varphi^{3}$ are generally required to represent. For the sake of simplicity, the above equations are omitted and indicated by $\varphi$.

The representation of the damaged region in the image in the wavelet domain is the loss of transform coefficients of wavelet transform $X_{n 0}(x)$ in index region $\Omega^{\prime}$. That is, when $(j, k) \in \Omega^{\prime}$, the area represented by the coefficient $a_{j, k}$ is damaged. The image restoration work is transformed into the coefficient restoration of the damaged area.

According to the TV restoration algorithm, the wavelet restoration model based on the wavelet domain can be expressed as:

$$
\min F\left(X_{n 0}, z\right)=\iint_{R^{2}}\left|\nabla_{x} X_{n 0}(\beta, x)\right| d x=T V\left(X_{n 0}(\beta, x)\right)
$$

In the equation, $X_{n 0}(\beta, x)$ has a wavelet transform $X_{n 0}(\beta, x)=T V\left(X_{n 0}(\beta, x)\right)$.

Combining the above, the full-variation curvature diffusion restoration algorithm of the wavelet domain can be obtained by using equation (23). Finally, by using the TVCDD algorithm, the restored image $Y_{n}$ of the structural part can be obtained.

\subsubsection{Restoration of texture parts}

Most texture restoration algorithms only consider either the global or local information of the image, which will result in the final repair effect only related to the information around the damaged area: however the global grasp is poor, or it only conforms to the global structure, so that the local restoration is rough. Xin proposed a Bayesian mean model based on compressed sensing sparse representation. The model proposed combining statistical theory with compressed-sensing reconstruction technology to make full use of image global and local information to restore damaged images. It can be seen from the above description that the restoration algorithm in this section uses the Bayesian mean model to restore the obtained texture part $X_{t}$.

By using the Bayesian mean model, it is possible to comprehensively utilize the local and global information in the image [10], and fully consider the influence of the image structure on the sample block selection when texture is used to fill the texture-damaged image $X_{t}$.

For the obtained texture image $X_{t}$, the damage area is set to $X_{t m}$. The basic idea of the Bayesian model is to use the competition model $\left\{M_{k}\right\}_{k=1}^{K}$ to describe the relationship between the damaged area $X_{t m}$ and the broken image $X_{t}$ :

$$
p\left[X_{t m} \mid X_{t}\right]=\sum_{k=1}^{K} a_{k} p\left(X_{t m} \mid X_{t}, M_{k}\right)
$$

competition between local and non-local, DCT and wavelet as well as other objectives with different properties in the same model.

In equation (24), the mean square error is introduced as:

$$
E\left[X_{t m} \mid X_{t}\right]=\sum_{k=1}^{K} a_{k} E\left(X_{t m} \mid X_{t}, M_{k}\right)
$$

Where $E\left[X_{t m} \mid X_{t}\right]$ is the minimum mean square error estimate of $M_{k}$ and the input image $X_{t}$ to be restored. In order to solve equation (25), the prior probability $a_{k}$ is first calculated.

According to the Bayesian equation, the prior probability of $M_{k}$ can be expressed using equation (26):

$$
p\left(M_{k} \mid X_{t}\right)=\frac{p\left(X_{t} \mid M_{k}\right) p\left(M_{k}\right)}{\sum_{I=1}^{K} p\left(X_{t} \mid M_{1}\right) p\left(M_{1}\right)}
$$

In the equation, $p\left(X_{t} \mid M_{k}\right)$ is a conditional probability and $p\left(M_{k}\right)$ is a probability function of $M_{k}$. In general, $p\left(M_{k}\right)$ satisfies the uniform distribution, and $p\left(X_{t} \mid M_{k}\right)$ satisfies the generalized Gaussian distribution. Then,

$$
a_{k}=\frac{p\left(X_{t} \mid M_{k}\right)}{\sum_{I=1}^{K} p\left(X_{t} \mid M_{1}\right)}
$$


Where $a_{k}$ is the scale and shape parameter of the generalized Gaussian distribution $M_{k}$. The texture restoration algorithm in this paper takes the standard Laplacian distribution $a_{k}=0$. The research of the traditional method proves that the standard Laplace can well approximate the coefficient of the high frequency band of the wavelet transform. For the Laplace distribution, the scale parameter $a_{k}$ can be expressed directly as:

$$
\widehat{a}=\frac{1}{N} \sum_{i=1}^{N}\left|X_{i}\right|
$$

In the equation, $\left|X_{i}\right|$ represents $N$ transformations on the sparse domain, so the calculation of the prior probability $a_{k}$ can be simplified as:

$$
a_{k}=\frac{\frac{1}{a_{k}}}{\sum_{I=1}^{K} \frac{1}{a_{k}}}
$$

It can be found from equation (29) that the scale parameter $a_{k}$ of $M_{k}$ affects the sparsity of the sparse representation. The smaller the scale factor is, the higher the sparsity based on the Bayesian model is; and the higher the sparsity is, the greater the weight in the mean calculation is. When the sparse representation is reconstructed, the global and local optimal solutions can be obtained according to the above-mentioned calculated sparsity as a weight in consideration of the update of the final result.

According to the Bayesian model described above, in the image restoration, the restoration algorithm can select the similarity closest to the $X$ texture pixels to fill according to the local feature and global structure of the image, and the clustering algorithm kNN.

When restoring the damaged image $X$ using local and non-local dictionaries, it is necessary to perform hard a threshold constraint on the sparse coefficients after the transformation.The sparse representation of the image is as follows:

$$
\min \|a\|_{0} s, t, X_{t}=D a
$$

Where $D$ is the corresponding sparse dictionary or transform, $a$ is the coefficient of the sparse representation, and the calculation of hard threshold constraint for $a$ is:

$$
a^{n+1}=H_{T}\left(a_{n}\right)
$$

In equation (31), the calculation rules for the thresholds $T$ and $H_{T}$ are as follows:

$$
H_{T}(x)=\left\{\begin{array}{l}
x,|x|>T \\
0,|x| \leq T
\end{array}\right.
$$

The operation of the restoration algorithm in the sparse domain can be expressed as:

$$
X_{t}^{\prime}=D^{H}\left[H_{T}\left(D X_{t}\right)\right]
$$

In equation (33), $D$ is a sparse dictionary calculated by the combination of equation (25) and equation (29), $D X_{t}$ is a sparse representation of the damaged image in the weighted sparse domain, $D^{H}$ is an inverse transformation of $D$, and $X_{t}^{\prime}$ is the final restoration image.

\subsection{Solution of compressed sensing image restoration algorithm based on improved SURF operator}

According to the spectral image complementation diagram, in the actual situation, due to the failure of satellite scan line corrector and other reasons, the obtained spectral image is a degraded spectral image with data loss, resulting in severe damage to the observation quality. To solve this problem, a complementary method that recovers potentially high-quality spectral images from degraded spectral images is particularly important. Before studying the specific complement method, the general mathematical model for the degradation of spectral image data loss is first given:

$$
y=H x+n
$$

In the above equation, $x$ represents the original highquality spectrum, $H$ represents the sampling operator of the analog data loss process, $y$ represents the low-quality spectral image after partial data loss, and $n$ represents the noise introduced during the sampling process.

In the spectral image complementation problem, it is known that there are low-quality spectral image $y$ with data-loss and sampling operator $H$, how to recover the original high-quality spectral image $x$. Since the image restoration problem is a typical ill-posed problem, it cannot be solved directly. Therefore, in the completion process, a priori knowledge of the spectral image needs to be introduced to impose a specific constraint on the solution space of the underdetermined problem. In the previous section, by analyzing the prior characteristics of spectral images, it is known that there are a large number of non-local self-similar 3D image blocks in the spectral image, and the matrix composed of these 3D image blocks with similar features has potential low rank characteristics. Based on this, it is proposed to introduce the low rank characteristics of the spectral image as a priori knowledge into the completion process, and obtain the following low rank optimization model based on the 3D image block:

$$
\begin{gathered}
\left\{\widehat{x}, \widehat{L}_{i}\right\}=\underset{x, L_{i}}{\arg \min } \sum_{i}\left\|\tilde{R}_{i} x-L_{i}\right\|_{F}^{2}+ \\
\tau \sum_{i} \operatorname{Rank}\left(L_{i}\right), \text { s.t }\|y-H x\|_{2}^{2} \leq \varepsilon
\end{gathered}
$$


Here, $\tilde{R}_{i} x=\left[R_{i 1} x, R_{i 2} x, \cdots, R_{i j} x\right] \in R^{s^{2 n \times l}}$ represents a matrix composed of the first $l$ images similar to the $i$ th 3D image block $x_{i}$. Each column in $\tilde{R}_{i} x$ represents a 3D image block with the length of $s^{2} n$ after stretching, $R_{i_{j}}, 1 \leq$ $j \leq 1$ represents a 3D block's operator, $\operatorname{Rank}\left(L_{i}\right)$ represents the rank of the matrix $L_{i}$ to be restored, that is, the number of non-zero singular values, and $\tau$ is the weight factor corresponding to a regular term $\sum_{i} \operatorname{Rank}\left(L_{i}\right)$. The minimum rank constraint for similar three-dimensional image blocks is achieved by minimizing regularity $\sum_{i} \operatorname{Rank}\left(L_{i}\right)$, and the allowed recovery error is controlled by $\varepsilon$.

In order to solve the problem, the constrained optimization problem in the above equation can be transformed into the unconstrained optimization problem in the following equation by introducing the Lagrangian multiplier:

$$
\begin{aligned}
& \left\{\hat{x}, \widehat{L}_{i}\right\}=\underset{x, L_{i}}{\arg \min }\|y-H x\|_{2^{+}}^{2} \\
& \eta \sum_{i}\left\|\tilde{R}_{i} x-L_{i}\right\|_{F}^{2}+\lambda \sum_{i} \operatorname{Rank}\left(L_{i}\right)
\end{aligned}
$$

Where, $\lambda$ and $\eta$ are two regular weighting factors obtained by normalizing the weight of the loyalty item $\mid y-$ $H x \|_{2}^{2}$. So far, a matrix low rank restoration model based on self-similar 3D image blocks has been obtained. However, since the matrix rank problem is an NP-hard combinatorial optimization issue, it is difficult to directly solve it. Candes et al. proved that the kernel norm of the matrix under certain conditions is equivalent to the convex approximation of the matrix rank issue. According to this theory, the following spectral image complementation model based on kernel norm's low rank approximation is obtained:

$$
\begin{aligned}
& \left\{\widehat{x}, \widehat{L}_{i}\right\}=\underset{x, L_{i}}{\arg \min }\|y-H x\|_{2}^{2}+ \\
& \eta \sum_{i}\left\|\tilde{R}_{i} x-L_{i}\right\|_{F}^{2}+\lambda \sum_{i}\left\|L_{i}\right\|
\end{aligned}
$$

Wherein, the kernel norm $\left|L_{i}\right| \mid$ represents the sum of all the singular values of the matrix $L_{i}$. The minimized objective function contains two unknown variables $x$ and $\left\{L_{i}\right\}$ to be solved, which are solved by an alternate optimization method. That is, when one variable in the low rank matrix $\left\{L_{i}\right\}$ and the spectral image $x$ are fixed, the other is solved; when a more precise single variable is obtained, the variable fixed in the previous step is inversely solved; thus the cycle is alternately optimized until convergence.

When the low rank matrix $\left\{L_{i}\right\}$ is fixed, the optimization model for solving the spectral image $x$ can be expressed as:

$$
x^{(k)}=\underset{x, L_{i}}{\arg \min }\|y-H x\|_{2}^{2}+\eta \sum_{i}\left\|\tilde{R}_{i} x-L_{i}\right\|_{F}^{2}
$$

$L_{i}^{(k)}$ and $x^{(k)}$ respectively represent low rank matrix and restored spectral image obtained after the $k$ th alternating optimization. The quadratic minimization problem in the above equation can be obtained by direct derivation to obtain the following closed-form solution:

$$
x^{(k)}=\left(H^{T} H+\eta \sum_{i} \tilde{R}_{i}^{T} \tilde{R}_{i}\right)^{-1}\left(H^{T} y+\eta \sum_{i} \tilde{R}_{i}^{T} L_{i}^{(k)}\right)
$$

In the actual solution, $\left(H^{T} H+\eta \sum_{i} \tilde{R}_{i}^{T} \tilde{R}_{i}\right)$ is a large matrix, and it is difficult to directly invert it. In order to reduce the computational complexity, we usually use the conjugate gradient algorithm to calculate the above equation.

When the spectral image $x^{(k)}$ is fixed, the optimization objective function for solving the low rank matrix $L_{i}$ is:

$$
L_{i}^{(k+1)}=\underset{L_{i}}{\arg \min } \sum_{i}\left\|\tilde{R}_{i} x-L_{i}\right\|_{F}^{2}+\frac{\lambda}{\eta} \sum_{i}\left\|L_{i}\right\|
$$

Compared with equation (40), it is not difficult to find that the optimization problem in the above equation can be solved by the singular value threshold (SVT) algorithm. The specific solution process is as follows:

$$
\left\{\begin{array}{c}
U^{(k+1)}, \sum^{(k+1 / 2)}, V^{(k+1)}=\operatorname{svd}\left(\tilde{R}_{i} x^{(k)}\right) \\
\sum^{(k+1)}=S_{\lambda(2 \eta)}\left(\sum^{(k+1 / 2)}\right) \\
L_{i}^{(k+1)}=U^{(K+1)} \sum^{(K+1)} V^{(K+1) T}
\end{array}\right.
$$

Where, $\operatorname{svd}\left(\tilde{R}_{i} x^{(k)}\right)$ represents singular value decomposition of matrix $\tilde{R}_{i} x^{(k)}, S_{\lambda(2 \eta)}\left(\sum^{(k+1 / 2)}\right)$ represents soft threshold value function, and the threshold value is $\lambda /(2 \eta)$.

Although the low-rank approximation model based on kernel norm is a convex optimization model, it is easier to solve than the original problem. However, the approximation of the original non-convex optimization problem with convex function is not accurate enough. In the sparse coding problem described above, the research results show that the non-convex $l_{p}(0<p<1)$ norm model can more accurately than the $l_{1}$ norm model to approximate the original $l_{1}$ norm optimization problem, and the recovered result has better sparsity. Inspired by this research, a nonconvex low rank approximation method is proposed. By using $\mid \alpha_{i} \|_{p}(0<p<1)$ as the nonconvex approximation of $\mid \alpha_{i} \|_{0}$, the accuracy of the model is further improved, and this spectral image complementation model based on nonconvex low rank approximation can be specifically equationted and expressed as:

$$
\left\{\widehat{x}, \widehat{L}_{i}\right\}=\underset{x, L_{i}}{\arg \min }\|y-H x\|_{2}^{2}+\eta \sum_{i}\left\|\tilde{R}_{i} x-L_{i}\right\|_{F}^{2}+\lambda \sum_{i}\left\|L_{i}\right\|_{p}
$$


Where $\sum_{i}\left\|L_{i}\right\|$ becomes the norm of matrix $L_{i}$, and $\alpha_{j}$ is the $j$ th singular value of matrix $L_{i}$. The model is similar to the above-mentioned solving method, and the spectral image $x$ and the low rank matrix $\left\{L_{i}\right\}$ are alternately solved by the alternating optimization method. Among them, $\left\{L_{i}\right\}$ is fixed to solve the optimal objective function of $x$ and its corresponding closed-form solution are as shown above, and the equation to solve the optimal objective function of $L_{i}$ by fixing $x$ is as follows:

$$
L_{i}^{(k+1)}=\arg \min \sum_{i}\left\|\tilde{R}_{i} x^{(k)}-L_{i}\right\|_{F}^{2}+\sum_{i}\left\|L_{i}\right\|_{P}
$$

Combining the idea of singular value decomposition and the generalized iterative enthalpy algorithm proposed by Zhang Lei et al. for solving non-convex sparse coding, the optimization model can be solved by the following equation:

$$
\left\{\begin{array}{c}
U^{(k+1)}, \sum^{(k+1 / 2)}, V^{(k+1)}=\operatorname{svd}\left(\tilde{R}_{i} x^{(k)}\right) \\
\sum^{(k+1)}=T^{G S T}\left(\sum^{(k+1 / 2)}\right) \\
L_{i}^{(k+1)}=U^{(K+1)} \sum^{(K+1)} V^{(K+1) T}
\end{array}\right.
$$

\section{Results}

In order to verify the effectiveness of the proposed SURF operator-based compressed sensing image restoration algorithm, an experiment is needed, and the experimental environment is Windows 8 operating system. The hardware configuration is $2.69 \mathrm{GHZ}$ dual-core Core processor with 16 GB memory. The proposed algorithm is compared with the compressed sensing image restoration algorithm based on rank minimization and the image fusion algorithm based on non-downsampling dual-tree complex contour transform and compressed-sensing pulsecoupled neural network. The experimental results are shown below.

The proposed algorithm is combined with the compressed sensing image restoration algorithm based on rank minimization and the image fusion algorithm based on non-downsampling dual-tree complex contour transform and compressed-sensing pulse-coupled neural network to make the experiment. The compressed sensing image restoration performance of each method is compared. The experiment compares the image restoration performance of three compressed sensing image restoration algorithms with different similar block sizes and the image restoration performance when the number of similar blocks is different. In the experimental results, A, B, and $\mathrm{C}$ are used to represent the compressed sensing image restoration algorithm based on rank minimization, the image fusion algorithm based on non-downsampling dualtree complex contour transform and compressed-sensing pulse-coupled neural network, and the proposed algorithm.

Image restoration of three image restoration algorithms at similar block sizes.

In general, the smaller the image block is, the more similar blocks are, but in the similar block search process, the Euclidean distance does not accurately measure the similarity. The image grouping composed of non-similar image blocks will reduce the sparsity, and affect image reconstruction performance, in addition, the smaller the image block is, the higher the complexity of similar block search is. In the experiment, three similar block sizes of $4 \times 4,8 \times 8$ and $16 \times 16$ are selected for the evaluation of image restoration performance. In order to increase the credibility of the experimental results, two different images are selected for experiment. The experimental results are shown in the Table 1 and Table 2.

Analysis of Table 1 and Table 2 data shows that the recovery performance of the three algorithms is different for different images, and the image restoration performance of each algorithm increases with the increase of sampling rate. At the same time, the proposed algorithm has excellent recovery performance for both images compared with the other two image restoration algorithms; When the sampling rate is the same, the image restoration performance of the proposed algorithm is the best, and the image restoration performance of the proposed algorithm is best when the similar block size is $4 \times 4$.

The proposed algorithm is compared with the image stabilization algorithm based on rank minimization and the image fusion algorithm based on non-downsampling double-tree complex contour transform and compressedsensing pulse coupled neural network. The image compression sensing reconstruction results are shown in Table 1. In order to simplify the description, in Table 1, the compressed sensing image restoration algorithm based on rank minimization is algorithm $\mathrm{A}$, image fusion algorithm based on non-downsampling dual-tree complex contour transform and compressed-sensing pulse-coupled neural network is proposed as algorithm 2, and the proposed algorithm is algorithm 3.

Analysis of Table 3 shows that the peak signal-to-noise ratio (SNR) of different methods for different spectral images is reconstructed, and different reconstruction results are bolded. From the average results of different methods, the peak-to-noise ratio of the algorithm A is only $22.61 \mathrm{~dB}$, 
Table 1: The recovery performance of three algorithms for compressed sensing image 1 under different similar block sizes

\begin{tabular}{lllll}
\hline Sampling Rate & Algorithm & $4 \times 4$ & $8 \times 8$ & $16 \times 16$ \\
\hline \multirow{3}{*}{0.3} & A & 36.72 & 36.33 & 36.27 \\
& B & 32.15 & 33.51 & 33.69 \\
0.4 & C & 31.42 & 32.07 & 33.12 \\
& A & 37.85 & 37.42 & 37.36 \\
& B & 34.47 & 35.64 & 35.65 \\
0.5 & C & 35.02 & 35.11 & 35.13 \\
& A & 38.61 & 38.24 & 38.15 \\
& B & 36.55 & 37.42 & 37.41 \\
0.6 & C & 36.41 & 37.33 & 37.42 \\
& A & 39.41 & 39.22 & 39.07 \\
& B & 38.31 & 38.12 & 38.19 \\
& C & 37.25 & 38.30 & 38.24 \\
\hline
\end{tabular}

Table 2: The recovery performance of three algorithms for compressed sensing image 2 under different similar block sizes

\begin{tabular}{lllll}
\hline Sampling Rate & Algorithm & $4 \times 4$ & $8 \times 8$ & $16 \times 16$ \\
\hline \multirow{2}{*}{0.3} & A & 28.02 & 27.51 & 27.35 \\
& B & 26.57 & 26.39 & 26.72 \\
& C & 26.41 & 26.52 & 26.47 \\
0.4 & A & 29.41 & 28.69 & 28.64 \\
& B & 28.51 & 27.75 & 27.92 \\
0.5 & C & 28.17 & 27.87 & 27.59 \\
& A & 30.17 & 29.82 & 30.10 \\
& B & 28.65 & 28.49 & 28.51 \\
0.6 & C & 28.34 & 28.13 & 28.47 \\
& A & 31.45 & 31.29 & 31.38 \\
& B & 29.41 & 29.72 & 29.69 \\
& C & 29.45 & 29.67 & 29.58 \\
\hline
\end{tabular}

Table 3: Comparison of Peak Signal-to-Noise Ratio of Spectral Image's Compressed Sensing Reconstruction Results by using Different Methods

\begin{tabular}{llll}
\hline Spectral image & Algorithm A & Algorithm B & Algorithm C \\
\hline City & $21.21 \mathrm{~dB}$ & $26.25 \mathrm{~dB}$ & $29.32 \mathrm{~dB}$ \\
Land & $24.12 \mathrm{~dB}$ & $30.21 \mathrm{~dB}$ & $32.03 \mathrm{~dB}$ \\
Indian & $20.25 \mathrm{~dB}$ & $30.48 \mathrm{~dB}$ & $30.12 \mathrm{~dB}$ \\
Toll & $24.87 \mathrm{~dB}$ & $26.47 \mathrm{~dB}$ & $32.63 \mathrm{~dB}$ \\
Mean value & $22.61 \mathrm{~dB}$ & $28.35 \mathrm{~dB}$ & $31.02 \mathrm{~dB}$ \\
\hline
\end{tabular}

and the algorithm B is followed and the proposed algorithm has the highest signal-to-noise ratio.

The proposed algorithm is compared with the image minimization-based compressed sensing image restoration algorithm and the image fusion algorithm based on non-downsampling dual-tree complex contour transform and compressed-sensing pulse-coupled neural network in the image restoration time (s). The experimental re- sults are shown in Fig. 1. In Fig. 1, A is a compressed sensing image restoration algorithm based on rank minimization, B is an image fusion algorithm based on nondownsampling dual-tree complex contour transform and compressed-sensing pulse-coupled neural network, and C is the proposed algorithm.

Analysis of Fig. 1 shows that the recovery time increases with the increase of the number of images. When 


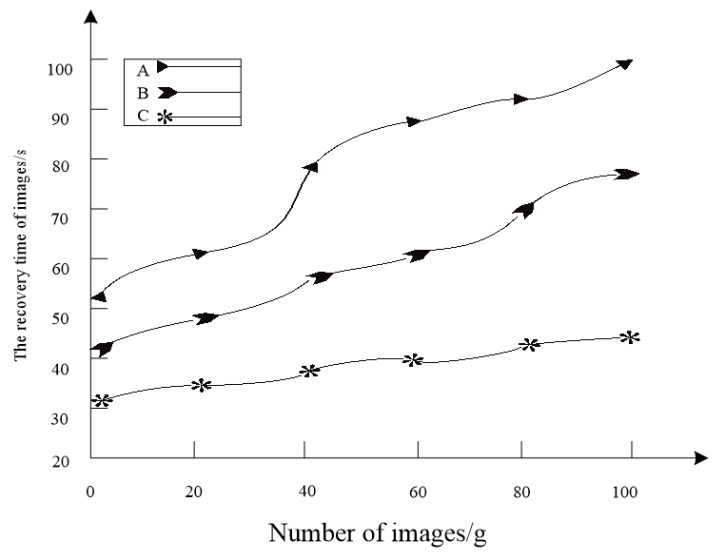

Figure 1: Comparison of image restoration time in different methods

the number of images is 20, the recovery time of algorithm $A$ is $62 \mathrm{~s}$, the recovery time of algorithm B is $48 \mathrm{~s}$, and the image restoration time of the proposed algorithm is $32 \mathrm{~s}$. It can be seen from the comparison that the algorithm $A$ and algorithm $B$ have different image restoration times from the propose algorithm by $30 \mathrm{~s}$ and $16 \mathrm{~s}$ respectively. When the number of images is 60 , the recovery time of algorithm A is $88 \mathrm{~s}$, the recovery time of algorithm B is $59 \mathrm{~s}$, and the image restoration time of the proposed algorithm is $36 \mathrm{~s}$. It can be seen from the comparison that the algorithm $A$ and algorithm $B$ have different image restoration times from the propose algorithm by $52 \mathrm{~s}$ and $23 \mathrm{~s}$ respectively. When the number of images is 100 , the recovery time of algorithm $A$ is $101 \mathrm{~s}$, the recovery time of algorithm $B$ is $78 \mathrm{~s}$, and the image restoration time of the proposed algorithm is $41 \mathrm{~s}$.

\section{Discussion}

The comparison shows that the compressed sensing image restoration algorithm based on rank minimization, and the image fusion algorithm based on non-downsampling dual-tree complex contour transform and compressedsensing pulse-coupled neural network have different image restoration time from the proposed algorithm by $80 \mathrm{~s}$ and $37 \mathrm{~s}$ respectively. It shows that the image reconstructed by the proposed algorithm has more detailed edges and texture structure, and the visual effect is better.

\section{Conclusions}

Image restoration is a process of reducing information loss caused by image damage by filling the damaged area in- formation and uninterrupted area information in the image. Digital image restoration is one of the hot research directions in the field of image processing. As a novel signal processing technology, compressed sensing has attracted the attention of researchers in various fields. Among them, the sparse representation and signal reconstruction techniques in compressed sensing have been widely used in the field of image restoration.

This paper proposes a compressed sensing image restoration algorithm based on improved SURF operator. The details are as follows:

The SURF feature vector set of the image is extracted, the vector set data is reduced into a single highdimensional feature vector by using a histogram algorithm, and the image HSV color histogram is extracted.

The MSA image decomposition algorithm is used to obtain the sparse representation of the image feature vector.For the data smoothing feature of the image structure part and the local similarity feature of the texture part, the total variation curvature diffusion method and the Bayesian weighting method are used in these two parts respectively to restore the image and combine the two parts.

The compressed sensing image restoration model is obtained by using Schatten-p norm. The image color complement is applied to the model. The compressed sensing image is solved iteratively by the alternating optimization method. The experimental results show that the proposed algorithm has better restoration performance, the restored image has finer edge and texture structure, and the visual effect is better.

Acknowledgement: 1. Hunan Provincial Natural Science Foundation of China: 'Development of New Automatic Test Equipment for Car Starter'(NO. 2014GK3054);

2. Hunan Provincial Education Science Foundation of China: 'Practice Research on Individualized Teaching Mode of Higher Vocational Education Based on Adaptive Learning' (NO. XJK18BXX006).

\section{References}

[1] Ahn J.H., Compressive Sensing and Recovery for Binary Images, IEEE Trans. Image Proc., 2016, 25(10), 4796-4802.

[2] Duan J.Z., Tang R.J., Deng H.M., Image Compression and Reconstruction based on Improved BP Neural Network and Tabu Table, Automat. Instrument., 2017 (3), 21-23.

[3] Elleuch I., Abdelkefi F., Siala M., Hamila R., Al-Dahir N., Robust gnc Approach for Quantised Compressed Sensing, Electr. Let., 2017, 53(19), 1306-1308.

[4] Hou Y., Wang X., Liu S., A Multiple Features Video Copy Detection Algorithm based on a Surf Descriptor, J. Inform. Proc. Syst., 
2016, 12(3), 502-510.

[5] Jalali S., Poor H.V., Universal Compressed Sensing for Almost Lossless Recovery, IEEE Trans. Inform. Theory, 2017, 63(5), 29332953.

[6] Kabbai L., Abdellaoui M., Douik A., Hybrid Local and Global Descriptor Enhanced with Colour Information, LET Image Proc., 2016, 11(2), 109-117.

[7] Meena V., Abhilash G., Robust Recovery Algorithm for Com pressed Sensing in the Presence of Noise, LET Sign. Proc., 2016, 10(3), 227-236.

[8] Meza P., Ortiz I., Vera E., Martinez J., Compressive Hyperspectral Imaging Recovery by Spatial-spectral non-local Means Regularization, Optics Expr., 2018, 26(6), 7043-7047.

[9] Shen Y.F., Zhu Z.M., Zhang Y.D. et al., Compressed Sensing Image Reconstruction Algorithm Based on Rank Minimization, Acta Electr. Sin., 2016, 44(3), 572-579.

[10] Wang C.H., Han D., An Effective Blind Forensic Detection Scheme for Median Filter Image, J. Chinese Acad. Electr. Sci., 2017, (6), 668-674.

[11] Wang S.M., Cheng B.L., Ye J.H., Wang M.W., Feature Extraction Method based on Weighted Multi-scale Zhang Quantum space, Data Acq. Proc., 2016, 31(4), 791-798.

[12] Yan N., Liao M., Jung C., Super-speed up Robust Features Image Geometrical Registration Algorithm, IET Image Proc., 2017, 10(11), 848-864.

[13] Yang J.R., Wu S., Wang H., Li J., Statistical Prior based Low Complexity Recovery for Compressed Image Sensing, IEEE/ACM Trans. Net., 2016, 24(1), 259-271.
[14] Yin M., Pang J.Y., Wei Y.Y. et al., Image Fusion Algorithm Combining NSDTCT and Compressed Sensing PCNN, J. Comp.-Aided Design \& Comp. Graph., 2016, 28(3), 411- 419.

[15] Zhang H., Li Z., Fast Stereo Matching based on Probability Assignment and Orb Operator, J. Inter. Math., 2016, 19(1), 129-145.

[16] Gao W., Wang W.F., The Fifth Geometric-Arithmetic Index of Bridge Graph and Carbon Nanocones, J. Diff. Eq. Appl., 2017, 23(1-2SI), 100-109.

[17] Lampart M., Zapomel J., Vibrations Attenuation of a System Excited by Unbalance and the Ground Movement by an Impact Element, Appl. Math. Nonlin. Sci., 2016, 1(2), 603-616.

[18] Serment Guerrero J.H., Lara Rivera E.A., Becerril Varela K., Suarez Contreras S., Ramirez Duran N., Detection and Insulation of Exo-Electrogen Microorganisms From Lodos Del Rio Lerma, State of Mexico, Mexico, Rev. Inter. Contamin. Ambient., 2017, 33(4), 617-628.

[19] Erat O., Isop W.A., Kalkofen D. et al., Drone-Augmented Human Vision: Exocentric Control for Drones Exploring Hidden Areas, J. IEEE Trans. Vis. Comp. Graph., 2018, 24(4), 1437-1446.

[20] Amat S., Busquier S., After Notes on Chebyshev's Iterative Method, Appl. Math. Nonlin. Sci., 2017, 2(3), 1-12.

[21] PengW., Ge S., Ebadi A.G., Hisoriev H., Esfahani M.J., Syngas Production by Catalytic Co-Gasification of Coal-Biomass Blends in a Circulating Fluidized Bed Gasifier, J. Clean. Prod., 2017, 168, 1513-1517. 